\title{
Gallium arsenide waveguides as a platform for direct mid-infrared vibrational spectroscopy
}

\author{
Julian Haas ${ }^{1} \cdot$ Robert Stach $^{1} \cdot$ Claudia Kolm ${ }^{2} \cdot$ Rudolf Krska $^{2,3} \cdot$ Boris Mizaikoff $^{1}$ \\ Received: 16 December 2019 / Revised: 16 February 2020 / Accepted: 24 February 2020 / Published online: 31 March 2020 \\ (C) The Author(s) 2020
}

\begin{abstract}
During recent years, mid-infrared (MIR) spectroscopy has matured into a versatile and powerful sensing tool for a wide variety of analytical sensing tasks. Attenuated total reflection (ATR) techniques have gained increased interest due to their potential to perform non-destructive sensing tasks close to real time. In ATR, the essential component is the sampling interface, i.e., the ATR waveguide and its material properties interfacing the sample with the evanescent field ensuring efficient photon-molecule interaction. Gallium arsenide (GaAs) is a versatile alternative material vs. commonly used ATR waveguide materials including but not limited to silicon, zinc selenide, and diamond. GaAs-based internal reflection elements (IREs) are a new generation of semiconductor-based waveguides and are herein used for the first time in direct spectroscopic applications combined with conventional Fourier transform infrared (FT-IR) spectroscopy. Next to the characterization of the ATR waveguide, exemplary surface reactions were monitored, and trace-level analyte detection via signal amplification taking advantage of surface-enhanced infrared absorption (SEIRA) effects was demonstrated. As an example of real-world relevance, the mycotoxin aflatoxin B1 (AFB1) was used as a model analyte in food and feed safety analysis.
\end{abstract}

Keywords Gallium arsenide - Surface modification - Evanescent field absorption - Self-assembled monolayers · Surface-enhanced infrared absorption $\cdot$ Mid-infrared chem/biosensor

\section{Introduction}

Spectroscopy in the mid-infrared spectral region (MIR, 2.5$25 \mu \mathrm{m}$ ) has nowadays evolved into a routinely applied analytical tool. Inherently non-destructive analysis as well as wellpronounced fundamental rotational, vibrational, and rotovibrational molecular transitions in the MIR renders this technique suitable for a wide variety of analytical sensing tasks.

Published in the topical collection Advances in Direct Optical Detection with guest editors Antje J. Baeumner, Günter Gauglitz, and Jiri Homola.

Boris Mizaikoff

boris.mizaikoff@uni-ulm.de

1 Institute of Analytical and Bioanalytical Chemistry, Ulm University, Albert-Einstein-Allee 11, 89081 Ulm, Germany

2 Institute of Bioanalytics and Agro-Metabolomics, Department of Agrobiotechnology (IFA-Tulln), University of Natural Resources and Life Sciences Vienna (BOKU), Konrad Lorenzstr. 20, 3430 Tulln, Austria

3 Institute for Global Food Security, School of Biological Sciences, Queens University Belfast, University Road, Belfast, Northern Ireland BT7 1NN, UK
Especially, attenuated total reflection (ATR) techniques have become increasingly popular for probing samples opaque in the MIR such as (bio)medical specimen within aqueous matrices. ATR sensing is based on the fundamental phenomenon of total internal reflection within a high-refractive-index waveguide material. Upon reflection at the analyte/internal reflection element (IRE) interface, an intensity exponentially decaying evanescent field is reaching into the adjacent media, which allows analyte interaction (e.g., via absorption) and the generation of ATR spectra. The penetration depth $d_{\mathrm{p}}$ of the evanescent field reaches to a distance of approx. 1/10 of the deployed wavelength $\lambda$ into the adjacent media, as described via Eq. (1).

$$
d_{\mathrm{p}}=\frac{\lambda}{2 \pi n_{1} \sqrt{\sin ^{2}(\theta)-\left(\frac{n_{2}}{n_{1}}\right)^{2}}}
$$

As evident from Eq. (1), the refractive indices of the ATR waveguide $n_{1}$ and the sample $n_{2}$ along with the angle of incidence $\theta$ are critical parameters, which are determining the sample interaction volume and the related detection 
capabilities besides the deployed wavelength. However, a limited set of IRE materials are suitable for ATR sensing in the MIR [1]. Among the routinely used IRE waveguide materials are silicon $(\mathrm{Si})$, zinc selenide $(\mathrm{ZnSe})$, germanium $(\mathrm{Ge})$, and diamond, whereas selection of the most suitable IRE waveguide is dependent on the anticipated field of application. In the present study, we demonstrate the utility of gallium arsenide (GaAs) as a promising alternative in the context of biorecognition sensing schemes. III/V semiconductor materials such as GaAs are well known in electronic circuit design for high-frequency data transfer, and are readily structured after epitaxial growth via wet and dry etching protocols. For photonic sensing devices, different structural sensing architectures based on thin-film GaAs waveguide technology have been recently pioneered by the research team of Mizaikoff and collaborators. However, to date, such sensing concepts including slab waveguides [2-4] and Mach-Zehnder interferometers (MZI) have been combined with cascade laser light sources (i.e., quantum and interband cascade lasers [QCLs, ICLs]) rather than broadband infrared spectrometers [5-7]. Hence, the present study for the first time combines GaAs waveguides with Fourier transform infrared (FT-IR) spectroscopy facilitating a more ubiquitous usage of this new generation of waveguide materials.

Waveguide-based sensor devices may in addition benefit from surface modification strategies for enhancing the sensor response leading to improved limits of detection (LODs). Exemplarily, analyte enrichment at the sensor surface and within the penetration depth of the evanescent field may be performed via chemical strategies such as preconcentration membranes, or biorecognition approaches such as immobilized enzymes, antibodies, or aptamers. In addition, the sensor robustness may be improved by protecting the IRE surface against, e.g., chemical degradation with selfassembled monolayers (SAMs) as routinely employed architectural motive [8]. Thiol-terminated long-chained organic molecules are in fact ideally suited for GaAs surface modification, as - similarly to gold - thiol anchoring groups also strongly bind to GaAs $[9,10]$. Bifunctional SAMs allow for further chemical modification after attachment of a first monolayer $[5,11]$. It should be noted though that passivating native oxide layers have to be removed from the GaAs surface prior to any immobilization scheme [12].

Due to similar binding chemistries, the immobilization of gold nanoparticles (AuNPs) at the GaAs waveguide surface is facilitated enabling additional amplification of the analytical signal via surface-enhanced infrared absorption (SEIRA) spectroscopy $[13,14]$. Thereby, signal amplification in ATRbased sensors for probing, e.g., small molecules such as pnitro benzoic acid or biologically relevant molecules including DNA or membrane proteins has been demonstrated [15-23]. Commonly, spherical nanoparticle structures are used in surface-enhanced spectroscopies. More recently, alternative geometries including plasmonic nanostars have demonstrated superior signal amplification in MIR sensing scenarios [24].

Given the anticipated increase in sensitivity, the present study has focused on the potential detection of mycotoxins, which have to be reliably detected in feed and food matrices such as corn, rice, maize, and milk at ppm-ppb concentration levels, as mandated by the respective authorities [25-27]. As a relevant example of such secondary fungus metabolites, aflatoxin B1 (AFB1) has been selected, which is considered among the most carcinogenic herbal substances known [28]. Conventionally, analytical techniques based on HPLC, LCMS, or GC/MS-MS are utilized for molecular discrimination of food pathogens [29]. However, such methods are time and cost consuming and are usually limited in portability. Alternatively, MIR-based sensing systems are promising device platforms for monitoring scenarios, albeit not competitive in molecular discrimination and sensitivity vs. the formerly mentioned laboratory techniques. In addition, MIR-based sensors may be significantly reduced in size towards highly integrated portable devices utilizing integrated photonics with a common material system such as GaAs for light source, detector, and waveguide sensing interface. While the direct detection of mycotoxins may be limited with ATR-based IR sensors [30-32], the evaluation of matrix changes resulting from fungal activity after infection during growth, harvest, or storage scenarios may be promising. As previously shown, matrix changes are indeed correlated with the presence of mycotoxins, as shown via FT-IR- and QCL-based techniques with sensitivities at EU regulatory limits [33-38]. However, utilizing broadband spectra of selected mycotoxins along with smart extraction schemes may increase the utility of MIR monitoring systems, as shown for the example of AFB1.

\section{Experimental section}

\section{Chemicals and materials}

Sodium acetate $\left(\mathrm{C}_{2} \mathrm{H}_{3} \mathrm{NaO}_{2}\right.$, water free), gold (III) chloride trihydrate $\left(\mathrm{HAuCl}_{4} \times 3 \mathrm{H}_{2} \mathrm{O}\right)$, trisodium citrate dihydrate $\left(\mathrm{C}_{6} \mathrm{H}_{5} \mathrm{O}_{7} \mathrm{Na}_{3} \times 2 \mathrm{H}_{2} \mathrm{O}\right)$, concentrated hydrochloric acid $(\mathrm{HCl}$, $32 \%), \mathrm{L}(+)$-ascorbic acid (AA), silver nitrate $\left(\mathrm{AgNO}_{3}\right)$, and sodium hypochlorite $\left(\mathrm{NaClO}, 14 \% \mathrm{Cl}_{2}\right.$ in aqueous solution) were purchased from Sigma-Aldrich. HS-PEG-SH (MW, 2000) was purchased from Rapp Polymere GmbH (Tübingen, Germany). Aflatoxin B1 was purchased from Acros Organics (NJ, USA). Absolute ethanol (EtOH), methanol $(\mathrm{MeOH})$, chloroform $\left(\mathrm{CHCl}_{3}\right)$, acetonitrile $(\mathrm{ACN})$, acetone (Propan-2-one), and isopropyl alcohol (IPA) were purchased from VWR. Milli-Q water $\left(10.8 \mathrm{M} \Omega\right.$ at $21{ }^{\circ} \mathrm{C}$, Millipore) was used in all experiments.

Commercially available diamond ATR crystals were purchased from Harrick Scientific (Harrick Scientific Products 
Inc., Pleasantville, NY, USA) and used without any further treatment. Si ATR crystals were laser cut from 3-in. wafers (crystal orientation $\langle 100>$, thickness $380 \pm 2 \mu \mathrm{m}$, prime FZ, undoped, double side polished (DSP), TTV $<10 \mu \mathrm{m}$, resistivity $10 \mathrm{k}-100 \mathrm{k} \Omega \mathrm{cm}$; Microchemicals $\mathrm{GmbH}$, Ulm, Germany). Debris from the cutting process was removed via subsequent sonication in acetone, IPA, and water. Further cleaning was performed according the RCA 1 and RCA 2 protocols to remove possible metal contaminations and to degrease the surface $[39,40]$. Furthermore, this treatment ensured a uniform oxide layer on the silicon crystal surface. GaAs ATR crystals were laser-cut from 2-in. wafers (crystal orientation $<100\rangle$, thickness $300 \mu \mathrm{m}$, undoped, DSP, resistivity $2 \mathrm{M} \Omega \mathrm{cm}$; AXT Europe, GEO Semiconductor Ltd., Geneva, Switzerland). Debris from the cutting process was removed as for the silicon crystals. Subsequent immersion in concentrated $\mathrm{HCl}(32 \%)$ and rinsing in water with subsequent drying in a nitrogen stream further removed residual debris and ensured growth of a thin and uniform native oxide layer on the GaAs crystal surface.

Basic material characteristics of the utilized ATR crystals are summarized in Table 1. Apart from the optical properties, such as the refractive index and the utilizable wavelength range, physical resilience properties additionally affect usage of the individual waveguide materials. As diamond is predominant for sensing in harsh and abrasive scenarios, suitable waveguides for chem/biosensing of aqueous samples are mainly governed by the accessible $\mathrm{pH}$ range.

\section{Instrumentation}

A Bruker Vertex 70 (Bruker Optics, Ettlingen, Germany) FTIR spectrometer, equipped with a BioATR II cell (Bruker Optics, Ettlingen, Germany) and a liquid nitrogen $\left(\mathrm{LN}_{2}\right)_{-}$ cooled mercury cadmium telluride (MCT) detector (Bruker Optics, Ettlingen, Germany) were used for comparative studies of the three selected ATR crystal materials. Spectra were recorded at a spectral resolution of $2 \mathrm{~cm}^{-1}$, averaging 200 scans each spectrum. Data acquisition was performed with the OPUS 6.5 software package (Bruker Optics, Ettlingen, Germany) and the Essential FT-IR spectroscopy toolbox (Operant LLC, USA). Scanning electron microscopy (SEM) images were acquired with a Helios NanoLab 600 scanning electron microscope (FEI, Eindhoven, The Netherlands). SEM image evaluation was performed with Fiji (distributions of ImageJ) [41]. XPS spectra were recorded with a XPS spectrometer from Physical Electronics (PHI 5800). Chemical structures were drawn with ChemBioDraw (Version 14.0.0.118, Ultra-Package, Perkin Elmer, Waltham, USA).

\section{Material performance}

The BioATR II cell optical setup is based on a two-crystal design with a zinc selenide ( $\mathrm{ZnSe}$ ) focusing crystal and a second, round crystal plate that is in direct contact with the sample. The design of the BioATR II cell enabled application of a high contact pressure between the $\mathrm{ZnSe}$ focusing crystal and the IRE, thus ensuring close contact between the two planar crystal surfaces and efficient direct optical coupling. Any use of a fluid in-between the two crystals was deliberately avoided to suppress unwanted absorbance features of such a fluid. Internal reflections within the ATR crystal depend on the thickness and the angle of incidence that are given by the material characteristic refractive index $n_{1}$. Homemade lasercut ATR disks were undoped to maintain transparency in the MIR, since doping drastically reduces the propagation efficiency via free carrier absorbance. Comparative performance tests were conducted with sodium acetate solutions of different concentrations as simple model analyte in aqueous solutions vs. water as background.

\section{Surface modification and synthesis of gold nanostars}

Gold nanostars (AuNSts) were produced via a modified procedure developed by Bibikova et al. [24]. In brief, NSts were fabricated via a modified seed-mediated growth protocol following Yuan et al. [42]. Nanoparticle seeds were prepared using the citrate reduction method introduced by Frens [43, 44]. For AuNSts synthesis, $300 \mathrm{~mL}$ of the seed solution was quickly added to $30 \mu \mathrm{L}$ of $1 \mathrm{M} \mathrm{HCl}$ and $10 \mathrm{~mL}$ of a $0.25 \mathrm{mM}$ $\mathrm{HAuCl}_{4}$ aqueous solution at room temperature under vigorous stirring. In a timely manner, $300 \mu \mathrm{L}$ of $2 \mathrm{mM} \mathrm{AgNO}_{3}$ and $150 \mathrm{~mL}$ of $0.1 \mathrm{M}$ AA were added simultaneously to the solution and the reaction solution was stirred for $30 \mathrm{~s}$ while its
Table 1 Material properties of the ATR waveguide materials investigated in the present study

\begin{tabular}{llllllll}
\hline Material & $\begin{array}{l}\text { Wafer } \\
\text { disk } \\
\text { thickness } \\
(\mu \mathrm{m})\end{array}$ & $\begin{array}{l}\text { Angle of } \\
\text { incidence } \\
\left({ }^{\circ}\right)\end{array}$ & $\begin{array}{l}\text { Internal } \\
\text { reflections }\end{array}$ & $\begin{array}{l}\text { Refractive } \\
\text { index } n \text { at } \\
1000 \mathrm{~cm}^{-1}\end{array}$ & $\begin{array}{l}\text { Utilizable } \\
\text { wavelength } \\
\text { range }\left(\mathrm{cm}^{-1}\right)\end{array}$ & $\begin{array}{l}\text { Penetration } \\
\text { depth at } \\
6 \mu \mathrm{m}(\mu \mathrm{m})\end{array}$ & $\begin{array}{l}\mathrm{pH} \\
\text { range }\end{array}$ \\
\hline Diamond & 250 & 45 & 10 & 2.4 & $4000-525$ & 0.70 & $1-14$ \\
GaAs & 300 & 30 & 6 & 3.3 & $10,000-667$ & 0.73 & $3-12$ \\
Silicon & 380 & 30 & 11 & 3.4 & $8900-1500$, & 0.70 & $1-12$ \\
\end{tabular}


color turned from light red to a grayish blue, indicating successful AuNSt synthesis.

Surface modification of the GaAs ATR crystal was conducted after mounting the precleaned GaAs ATR crystal disks into the BioATR II cell between the ZnSe focusing element and a PTFE sealing ring. Forty microliters auf conc. $\mathrm{HCl}(32 \%)$ was deposited on the GaAs surface for $60 \mathrm{~s}$ to remove the native oxide and thus create a clean and active $\mathrm{GaAs}$ surface. The $\mathrm{HCl}$ solution was subsequently removed by rinsing with about $10 \mathrm{~mL}$ dry and degassed ethanol. A small portion of the degassed ethanol was left on the chip surface to suppress regrowth of the native oxide. Forty microliters of a $2 \mathrm{mM} \alpha$ - $\omega$-dimercapto polyethylene glycol (SH-PEG-SH) ethanolic solution (dry and degassed with argon) was added into the sampling tray of the BioATR II cell. The thus prepared chips were immersed for $12 \mathrm{~h}$ and subsequently rinsed with approx. $50 \mathrm{~mL}$ ethanol to remove unbound physisorbed linker molecules. Afterwards, the chips were immersed for another $12 \mathrm{~h}$ in $40 \mu \mathrm{L}$ of the AuNSt suspension and subsequently rinsed with approx. $50 \mathrm{~mL} \mathrm{H}_{2} \mathrm{O}$, to remove unbound AuNSts from the surface. The surface was dried with a nitrogen stream afterwards. An exemplary workflow is shown in Fig. 1.

\section{Mycotoxin analysis}

Aflatoxin B1 (AFB1) was dissolved in a 2:1 mixture of methanol and chloroform to readily dissolve the $\mathrm{AFB} 1$. $\mathrm{CHCl}_{3}$ is known to stabilize dissolved AFB1 for shelf storage [45]. Methanol is a commonly utilized solvent for extraction purposes. The binary mixture was chosen to ensure complete dissolution of the AFB1 while keeping the consumption of chlorinated solvents at a moderate level. Acetonitrile, that is routinely used as solvent for mycotoxin standard preparation, was neglected since commercially available acetonitrile often contains relatively high levels of residuals after evaporation which can easily be confused with analyte signals and its nonneglectable toxicity due to metabolization to hydrogen cyanide. For AFB1 detection, $10 \mu \mathrm{L}$ of solutions with different concentrations was added into the sample tray of the BioATR II cell, with bare and modified (for SEIRAS experiments) GaAs chips mounted. The solvent was left to evaporate, which was complete after about $10 \mathrm{~min}$ at room temperature. After experiments, all surfaces were cleaned with sodium hypochlorite to detoxicate AFB1 [46].

\section{Results}

\section{ATR waveguide comparison}

Comparing the transmission windows of $\mathrm{Si}$, diamond, and GaAs in the MIR spectral region ranging from 4000 to $600 \mathrm{~cm}^{-1}$ indicates that GaAs possesses a transparency window throughout the entire analytically useful MIR spectral window (Fig. 2). Si provides comparable transmission properties at higher wavenumbers, yet is less transparent towards lower wavenumbers. Especially around $1200 \mathrm{~cm}^{-1}$, silicon oxide possesses a vibrational mode, and thus reduced transparency in the so-called molecular fingerprint region. Diamond provides high chemical and physical resilience and thus offers versatile options for applications in harsh sensing scenarios including elevated temperatures, strong acids and bases, and contact with abrasive samples. Around $2400 \mathrm{~cm}^{-1}$, phonons are excited in the crystal lattice of diamond, which limits the usage of diamond in this spectral regime to wavenumbers lower than $1700 \mathrm{~cm}^{-1}$. It should be noted that the single-channel spectra shown in Fig. 2 are superimposed by the device characteristics of the utilized FT-IR spectrometer (i.e., the instrument function), as well as atmospheric water vapor bands around $1600 \mathrm{~cm}^{-1}$ and $3700 \mathrm{~cm}^{-1}$ and carbon dioxide bands around $2400 \mathrm{~cm}^{-1}$. However, since all waveguide materials were studied in the same system and at the same conditions, a fair comparison is ensured.

To directly compare the performance of GaAs to the more routinely used ATR waveguide materials Si and diamond in an ATR-based sensing scheme, sodium acetate was selected as model analyte due to its high solubility in water, its non-toxicity, and its well-pronounced MIR fingerprint spectrum. Calibration functions were established via the integrated peak areas of two different absorbance bands of sodium acetate (Figs. 3 and 4). For the absorption band of the $\mathrm{CO}_{2}$ asymmetric stretch centered around $1550 \mathrm{~cm}^{-1}$, an integration range from 1600 to $1460 \mathrm{~cm}^{-1}$ was used. For the absorption band of the $\mathrm{CO}_{2}^{-}$symmetric stretch centered around $1413 \mathrm{~cm}^{-1}$, an integration range from 1458 to $1366 \mathrm{~cm}^{-1}$ was used. In both cases, diamond shows the steepest slope of the calibration function, which defines the sensitivity followed by $\mathrm{Si}$ and GaAs. Due to the wafer disk thickness of $300 \mu \mathrm{m}$, the GaAs disks support only 6 internal reflections, i.e., the least number of internal reflections of the three compared disks. Hence, in a

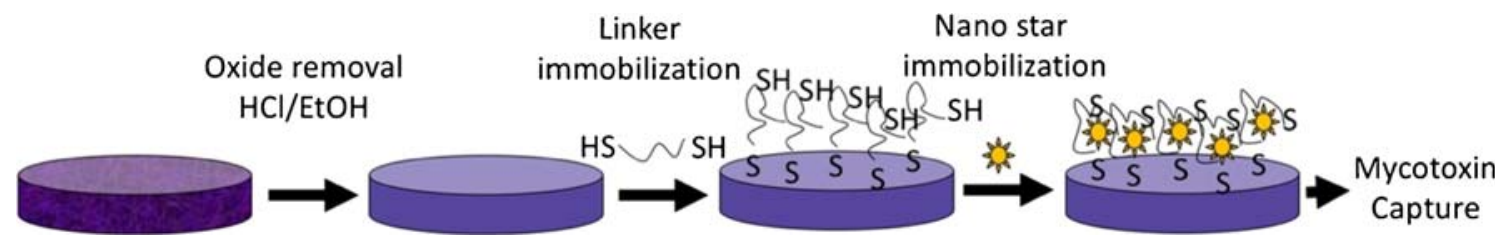

Fig. 1 Schematic workflow of the surface modification and subsequent AuNSt immobilization at GaAs ATR disk waveguides 


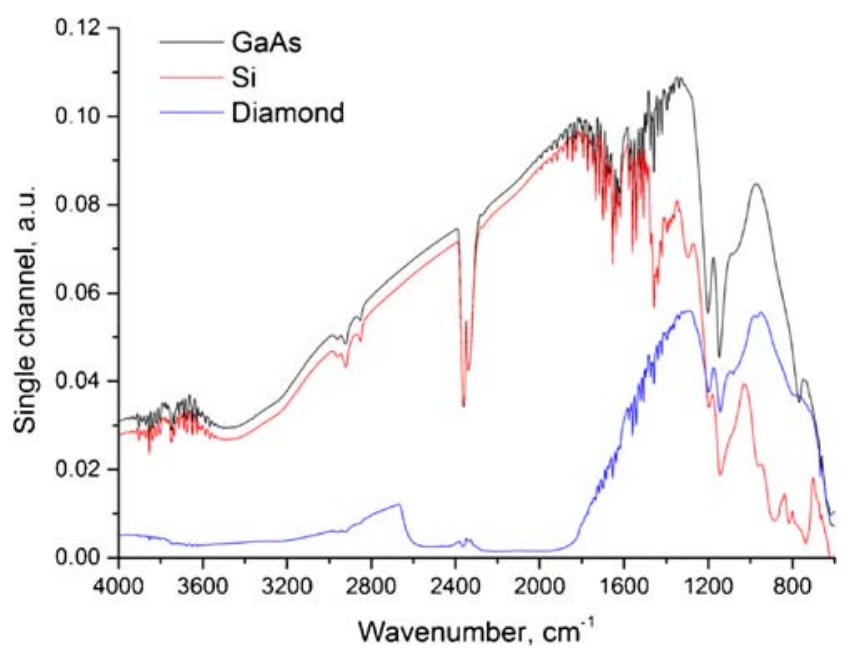

Fig. 2 Single-channel spectra of GaAs, $\mathrm{Si}$, and diamond ATR disk waveguides mounted in the same BioATR II cell

next step, the spectral response was normalized to the number of internal reflections by calculating the effectivity $E$ of an IRE as $E=$ sensitivity/noise, whereas the sensitivity is represented by the slope of the calibration function [47]. Consequently, the energy throughput (Fig. 2) is correlated with the spectral response, and the number of internal reflections is normalized. As shown in Table 2, the resulting normalized efficiency of GaAs ATR waveguides is approx. $40 \%$ higher than diamond rendering GaAs a versatile alternative to commonly utilized ATR waveguide materials, if sensitivity is the prime characteristic of interest.

Alternative to calculating the efficiency of the waveguide materials in direct comparison, the LOD as a common analytical figure-of-merit may be derived from the calibration functions shown in Figs. 3 and 4. The limit of detection was calculated according to DIN 32645 taking the $y$-axis intercept, the slope, the standard deviation, and significance level of $96 \%$ into account. The $y$-axis intercept has to be considered, as any instrument response due to noise- or non-analyterelated response leads to a potential offset of the calibration

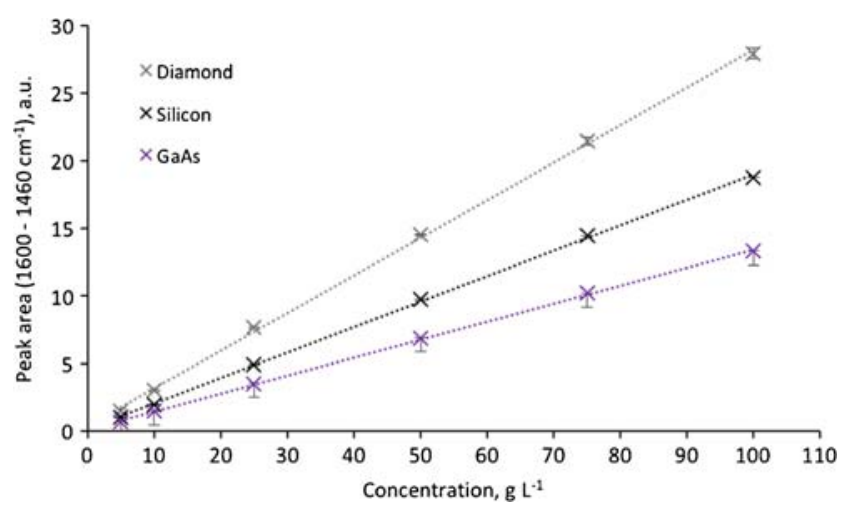

Fig. 3 Calibration functions for sodium acetate with ATR disk waveguides made from $\mathrm{GaAs}, \mathrm{Si}$, and diamond evaluating the absorbance band from 1600 to $1460 \mathrm{~cm}^{-1}$

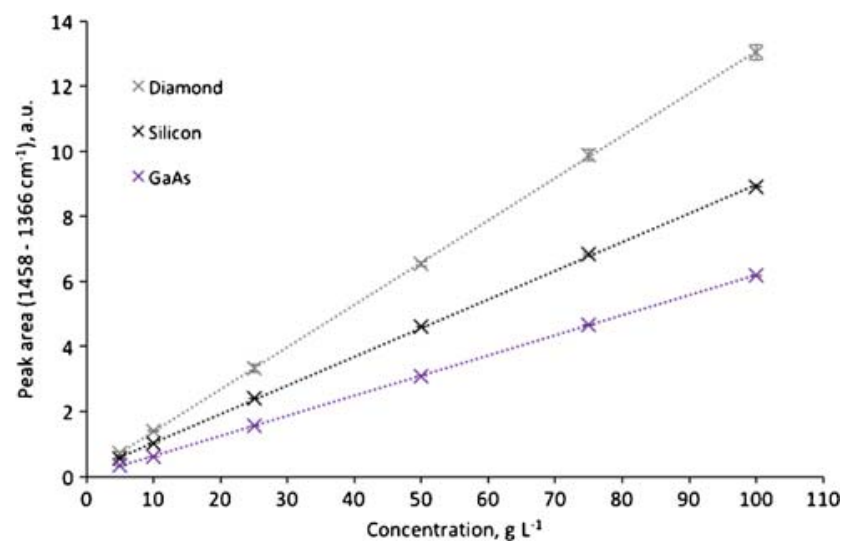

Fig. 4 Calibration functions for sodium acetate with ATR disk waveguides made from $\mathrm{GaAs}, \mathrm{Si}$, and diamond evaluating the absorbance band from 1458 to $1366 \mathrm{~cm}^{-1}$

function. As summarized in Table 3, all three materials provide LODs in the same concentration range with GaAs being slightly superior substantiating its utility as an alternative IRE waveguide material with similar sensor performance.

\section{GaAs surface modification and nanoparticle immobilization}

As a macroscopic tool to investigate surface modification procedures, water contact angle measurements were performed at different stages of the GaAs modification process. Etched GaAs showed a contact angle of $61^{\circ} \pm 7^{\circ}$ indicating a quite hydrophobic surface due to removal of surface oxide and hydroxide functional groups, as confirmed in literature [10]. The HS-PEG-SH functionalized GaAs surfaces lead to a contact angle of $19^{\circ} \pm 5^{\circ}$. These results are also in accordance with literature values for the functionalization of surfaces with SAMs via organo-dithioles, which are hydrophilic due to formation of hydrogen bonds with water molecules [48].

GaAs - as an ATR waveguide - enables direct in situ monitoring of surface modification protocols. To study the immobilization kinetics at GaAs in more detail, MIR spectra were recorded during the immobilization process of HS-PEG-SH at the deoxidized surface in situ via IR difference spectra. A background spectrum was recorded after depositing $40 \mu \mathrm{L}$ of the HS-PEG-SH solution in ethanol at the waveguide surface and closing the sampling chamber lid. Sample spectra

Table 2 Evaluation of noise and efficiency of the investigated diskshaped waveguide materials derived from the calibrations shown in Fig. 3

\begin{tabular}{llllll}
\hline Peak area: & \multicolumn{1}{l}{$1600-1460 \mathrm{~cm}^{-1}$} & & & \\
\cline { 2 - 4 } & Slope & Intercept & Standard deviation & Noise & Efficiency \\
\hline Diamond & 0.2784 & 0.3811 & 1.1557 & 0.0018 & 155 \\
Silicon & 0.1883 & 0.1597 & 0.7604 & 0.0020 & 94 \\
GaAs & 0.1328 & 0.1433 & 0.7108 & 0.0006 & 221 \\
\hline
\end{tabular}


Table 3 Comparison of the LOD for aqueous solutions of sodium acetate for two different absorbance bands using ATR waveguide disks made from $\mathrm{GaAs}, \mathrm{Si}$, and diamond

\begin{tabular}{lll}
\hline & \multicolumn{2}{l}{$\operatorname{LOD}\left(\mathrm{g} \mathrm{L}^{-1} ; \times 1000 \mathrm{ppm}\right)$} \\
\cline { 2 - 3 } & $\begin{array}{ll}1600- \\
1460 \mathrm{~cm}^{-1}\end{array}$ & $\begin{array}{l}1458- \\
1366 \mathrm{~cm}^{-1}\end{array}$ \\
\hline Diamond & 3.4 & 2.8 \\
Silicon & 2.2 & 1.8 \\
GaAs & 2.1 & 0.8 \\
\hline
\end{tabular}

were recorded subsequently after $10 \mathrm{~min}$ each for $7 \mathrm{~h}$. The C$\mathrm{O}-\mathrm{C}$ band of the HS-PEG-SH molecule was selected as the exemplary band and evaluated during the modification process at GaAs surface over time. For this purpose, the absorption band of the $\mathrm{C}-\mathrm{O}$ stretch vibration of the $\mathrm{C}-\mathrm{O}-\mathrm{C}$ ether functional group centered around $1134 \mathrm{~cm}^{-1}$ was integrated from 1180 to $1085 \mathrm{~cm}^{-1}$. As shown in Fig. 5, the band representing the C-O-C vibration strongly increases during the first hour of immersion, and afterwards increases according to a Freundlich isotherm-like behavior. Adsorption kinetics according to the Freundlich model assume a decreasing enthalpy of adsorption due to an increased surface coverage, and thus, non-equal adsorption places due to repulsion of the adsorbed molecules. Such a behavior may indeed be expected from the chemical and structural composition of the large HSPEG-SH molecules. Furthermore, complete surface coverage cannot be modeled with a Freundlich isotherm, which is in further accordance with the expected behavior of the HSPEG-SH adsorption kinetics.

Due to the length of the PEG back chain, potential bilayers or otherwise physisorbed HS-PEG-SH molecules within the

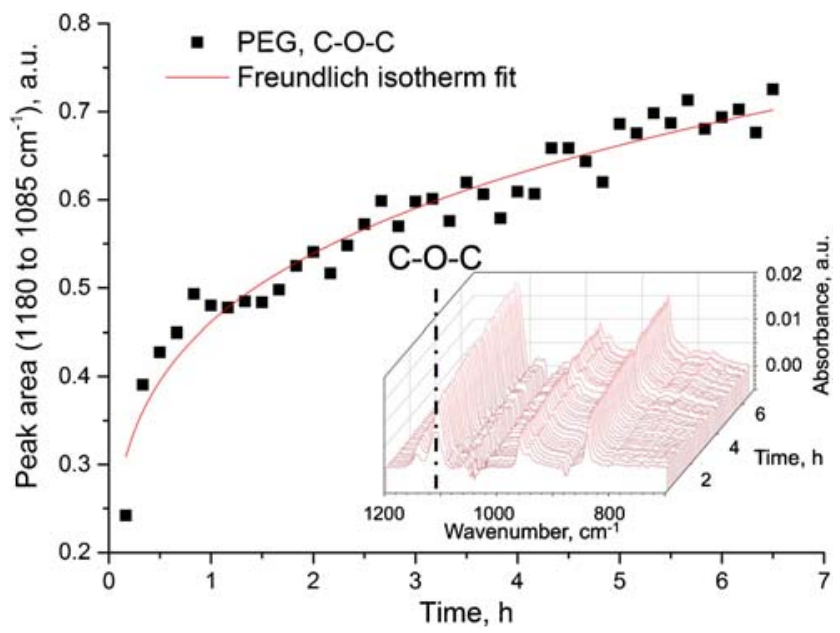

Fig. 5 IR spectral tracking of the C-O-C band of the HS-PEG-SH polymer during immobilization at the GaAs waveguide disk surface during the first $7 \mathrm{~h}$ of the immobilization. The Freundlich isotherm-like fit follows a relation according to $y=0.46 \cdot x^{1 / 4.46}$. The inset shows the timeresolved development of the IR spectra with the $\mathrm{C}-\mathrm{O}-\mathrm{C}$ band highlighted by a dashed line chemisorbed and strongly bound molecules are expected in close vicinity of the surface, and therefore within the penetration depth of the evanescent field. Hence, sufficient and thorough solvent rinsing after the immobilization procedure has to be ensured for removing non-covalently bound molecules at the waveguide surface.

To further evaluate the efficiency of the immobilization and AuNSt binding performance, SEM images and XPS spectra were recorded at the finally modified waveguide surface. The exemplary SEM image shown in Fig. 6 readily shows immobilized AuNSts at the HS-PEG-SH-modified surface after thorough rinsing with water and blow drying. Thus, the prepared AuNSts show a narrow size distribution at an average feret diameter of $55 \pm 30 \mathrm{~nm}$, which results from the seedmediated approach [24]. Furthermore, the AuNSt surface coverage is calculated at approx. $30 \%$, as derived from a representative area of approx. $1.5 \mu \mathrm{m}^{2}$. This uniformity is of importance to ensure reproducible surface coverage and consequently, reliable sensor response during repeated experiments.

Elemental surface coverage was evaluated via XPS studies addressing the atomic concentration at approx. 1-12 nm depth from the sample surface. An exemplary XP spectrum is shown in Fig. 7, and the derived atomic surface concentrations are summarized in Table 4.

$\mathrm{C}, \mathrm{O}, \mathrm{S}$, and $\mathrm{Au}$ introduced by modifying a GaAs surface with thiol-modified polyethylene glycol and subsequent gold nanostar immobilization are present in the XP spectra. The dominating atomic concentration of carbon of about $73 \%$ indicates possible shielding of the generated radiation from the other elements, since a ratio of approx. 2:1 for $\mathrm{C}: \mathrm{O}$ correlates to the PEG component.

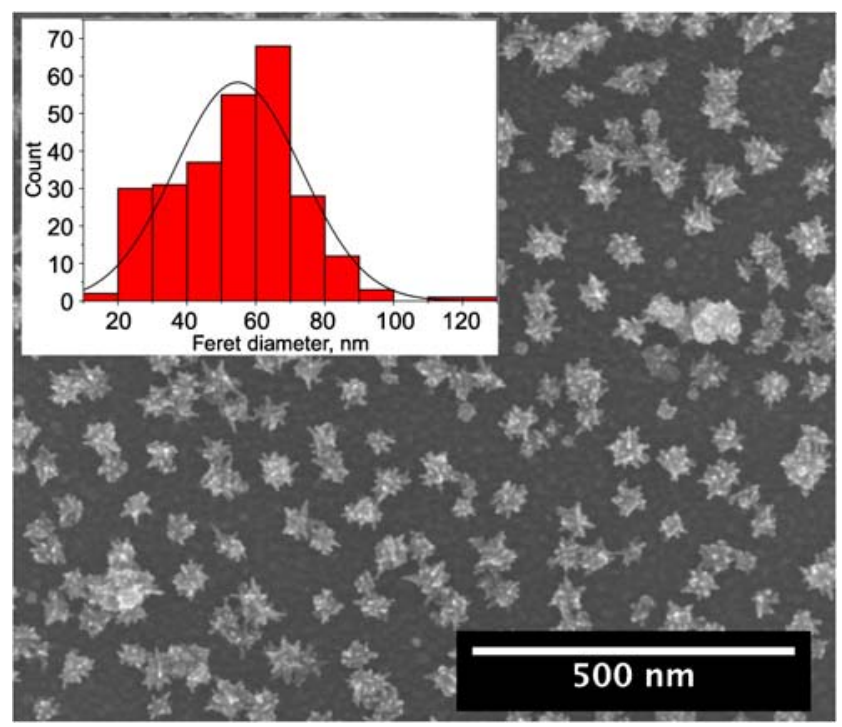

Fig. 6 Scanning electron microscope (SEM) image of a representative section of the immobilized AuNSts at the GaAs waveguide disk surface with an average feret diameter of $55 \mathrm{~nm}$ of the AuNSts 
Fig. 7 XPS spectrum of a HSPEG-SH-modified GaAs waveguide disk with immobilized AuNSts



\section{Direct broadband mycotoxin detection via SEIRA on $\mathrm{GaAs}$ waveguide disks}

Due to the signal amplifying SEIRA effect, low-level detection of relevant analytes may benefit in addition to chem/ biorecognition schemes from such signal enhancement strategies. Herein, the detection of trace levels of ABF1 was selected serving as a relevant candidate to evaluate potential benefits of HS-PEG-SH-modified ATR waveguide surfaces decorated in addition with AuNSts.

An exemplary IR-ATR spectrum of 600 ppm AFB1 after solvent evaporation is shown in Fig. 8. The IR fingerprint shows the anticipated complex absorbance pattern associated with large organic molecules comprising a variety of functional moieties, as present in AFB1. As GaAs is widely transparent in the fingerprint region, identification of pure (bio)molecules via unique MIR fingerprint spectra is readily achievable.

Comparative calibrations for the trace-level detection of AFB1 in the low ppm concentration regime with and without SEIRA-active substrate are shown in Fig. 9. To avoid interference with absorption bands of atmospheric water vapor, integration of an absorption band ranging from 1495 to $1425 \mathrm{~cm}^{-1}$ was selected to represent the AFB1 concentration. The SEIRA-active substrate shows an analytical signal amplification by a factor of approx. 4 vs. the non-enhanced counterpart. This enhancement factor is similar to previously reported enhancement factors for biomolecules [49]. This enhancement factor is clearly at the lower range of possible

Table 4 Atomic surface concentration derived from the XPS analysis $(N=2)$

\begin{tabular}{lllllll}
\hline Atom peak & C1s & O1s & S2p & Ga3d & As3d & Au4f \\
Atomic concentration (\%) & 73 & 4 & 11 & 6 & 5 & 1 \\
\hline
\end{tabular}

SEIRA enhancement factors reported in literature, where enhancement factors up to 1000 have been shown [50]. Potential strategies towards higher enhancement factors are increasing the surface coverage density of AuNSts [5]. The SEIRA calibration function shows a slight increase in slope, i.e., sensitivity by approx. $20 \%$. However, the corresponding standard deviations of the SEIRA-based calibration function appear elevated as well. This increased sample-to-sample variance may be attributed to minor differences between individual modified IRE surfaces.

Deriving the LOD of AFB1 at bare GaAs according to DIN 32645 , a value $4000 \mathrm{ppb}$ has been calculated. For the AuNStdecorated substrate, a slightly improved LOD of $3500 \mathrm{ppb}$ was resultant. The presented exemplary surface decoration motif led to an LOD enhancement of approx. 14\%. This improvement may be sufficient when working at regulatory limits for selected analytes boosting the performance by an



Fig. 8 IR-ATR spectrum of 600 ppm AFB1 after solvent evaporation ( $2 \mathrm{~cm}^{-1}$ spectral resolution; 200 scans averaged per spectrum) 
a

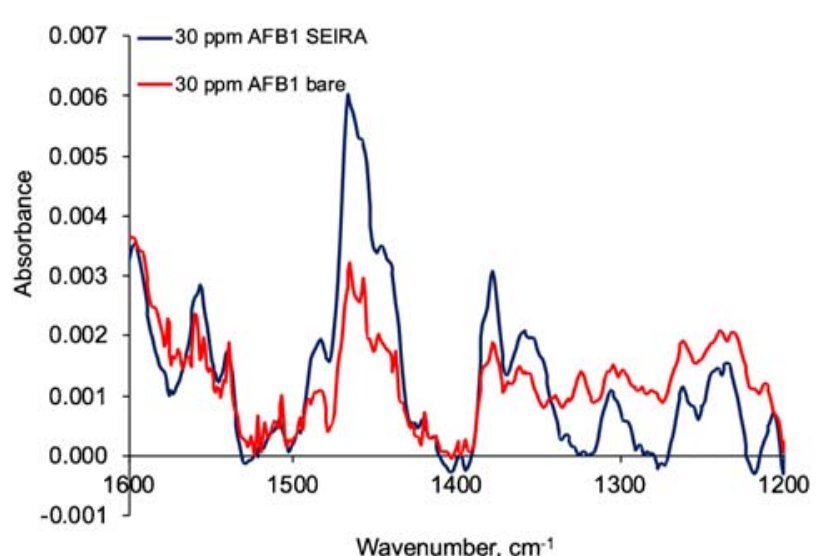

Fig. 9 a Raw spectra of AFB1 fingerprint spectra with a concentration of $30 \mathrm{ppm}$ on a bare substrate (red line) and on a modified SEIRA-active substrate (blue line). b Calibration functions of the spectral response of an

order of magnitude into a useful range. As an alternative or in addition, incorporating more sophisticated nanostructures giving rise to SEIRAS effects along with selective analyte capture and enrichment schemes is anticipated to further enhance future sensor performance. Likewise, more sophisticated chemometric strategies need to be applied in combination with such motifs to extract relevant analyte signals from more complex real-world analyte samples.

\section{Conclusions and outlook}

The implementation of GaAs as an internal total reflection waveguide for broadband IR ATR sensing concepts has been evaluated in detail and has been confirmed as a versatile alternative to conventionally used ATR waveguide materials such as Si or diamond. Similar to gold surfaces, GaAs semiconductors facilitate surface chemistries via covalent thiol bonds, thus enabling a wide variety of immobilization architectures based on this inherently robust surface modification towards chem/ biosensing interfaces. GaAs is a widely used semiconductor platform for thin-film on-chip waveguide architectures in photonic circuitries. Herein, we evaluate the utility of GaAs serving as an active sensing element along with potential surface modification strategies for the implementation of highly integrated photonic chem/biosensors at a chip scale based on a new semiconductor platform. As a result, IR light sources and detectors operating in the MIR that are based on GaAs architectures may now be fully integrated with chem/biodetection schemes using the same material as the optical transducer element facilitating evanescent field sensing. Using surfaceenhanced infrared effects enables the additional amplification of the obtained analytical signals. While thin-film waveguide technology based on GaAs architectures has already

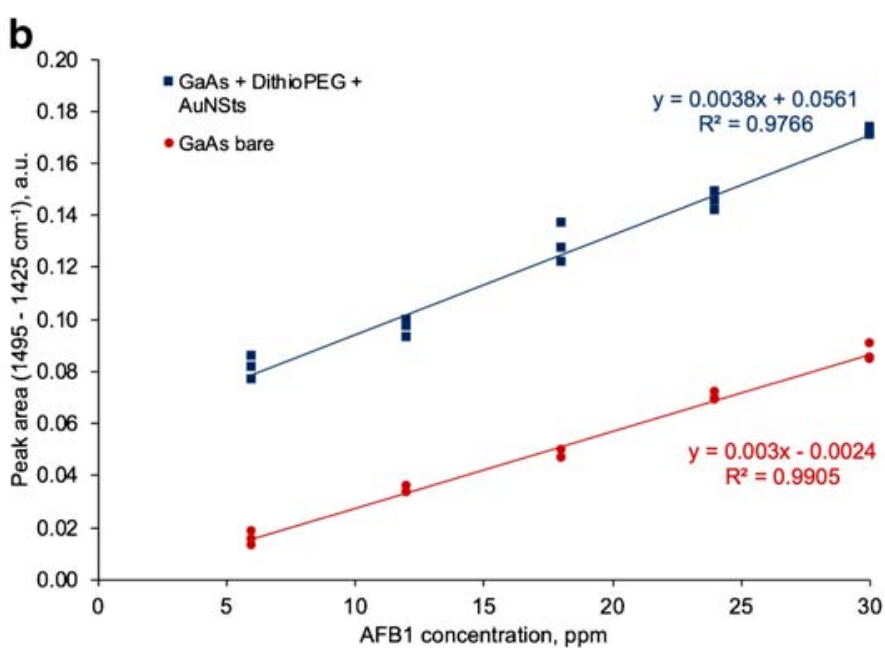

exemplary AFB1 band integrated from 1495 to $1425 \mathrm{~cm}^{-1}$ at a bare (red) vs. a AuNSt-modified GaAs waveguide disk surface (blue) $(N=3)$

demonstrated its utility, broadband direct spectroscopies may likewise benefit from advanced ATR waveguide designs and innovative waveguide materials $[51,52]$.

Acknowledgments T. Diemant at the Institute of Surface Chemistry and Catalysis (IOK) at Ulm University is thanked for support with XPS analysis. Furthermore, the machine shop of Ulm University is thanked for assistance in the laser cutting process. The Focused Ion Beam Center UUlm is thanked for support with electron microscopy imaging.

Funding information Open Access funding provided by Projekt DEAL. The authors received financial support from the Deutsche Forschungsgemeinschaft (DFG) under Sonderforschungsbereich/ Transregio (SFB/TRR) 234 "CataLight."

\section{Compliance with ethical standards}

Conflict of interest The authors declare that they have no conflicts of interest.

Open Access This article is licensed under a Creative Commons Attribution 4.0 International License, which permits use, sharing, adaptation, distribution and reproduction in any medium or format, as long as you give appropriate credit to the original author(s) and the source, provide a link to the Creative Commons licence, and indicate if changes were made. The images or other third party material in this article are included in the article's Creative Commons licence, unless indicated otherwise in a credit line to the material. If material is not included in the article's Creative Commons licence and your intended use is not permitted by statutory regulation or exceeds the permitted use, you will need to obtain permission directly from the copyright holder. To view a copy of this licence, visit http://creativecommons.org/licenses/by/4.0/.

\section{References}

1. Haas J, Mizaikoff B. Advances in mid-infrared spectroscopy for chemical analysis. Annu Rev Anal Chem. 2016;9:45-68. https:// doi.org/10.1146/annurev-anchem-071015-041507. 
2. Sieger M, Haas J, Jetter M, Michler P, Godejohann M, Mizaikoff B. Mid-infrared spectroscopy platform based on GaAs/AlGaAs thinfilm waveguides and quantum cascade lasers. Anal Chem. 2016;88: 2558-62. https://doi.org/10.1021/acs.analchem.5b04144.

3. Haas J, Stach R, Sieger M, Gashi Z, Godejohann M, Mizaikoff B. Sensing chlorinated hydrocarbons via miniaturized GaAs/AlGaAs thin-film waveguide flow cells coupled to quantum cascade lasers. Anal Methods. 2016;8:6602-6. https://doi.org/10.1039/ C6AY01450J.

4. Wang X, Sieger M, Mizaikoff B (2013) Toward on-chip mid-infrared chem/bio sensors using quantum cascade lasers and substrateintegrated semiconductor waveguides. In: Razeghi M (ed) Proc. SPIE. p 86312M.

5. Leidner L, Ewald M, Sieger M, Mizaikoff B, Gauglitz G. Migrating the Mach-Zehnder chemical and bio-sensor to the mid-infrared region. Proc SPIE. 2013;8774:87740S. https://doi.org/10.1117/12. 2017387.

6. Sieger M, Balluff F, Wang X, Kim S, Leidner L, Gauglitz G, et al. On-chip integrated mid-infrared GaAs/AlGaAs Mach-Zehnder interferometer. Anal Chem. 2013;85:3050-2. https://doi.org/10.1021/ ac302551s.

7. Haas J, Wilk A, Kokoric V, Mizaikoff B. Next-generation midinfrared chemical and biological sensors: combining quantum cascade lasers with thin-film and hollow waveguides. Spectrosc Online. 2017;32:22-30.

8. Kirchner C, George M, Stein B, Parak WJ, Gaub HE, Seitz M. Corrosion protection and long-term chemical functionalization of gallium arsenide in an aqueous environment. Adv Funct Mater. 2002;12:266. https://doi.org/10.1002/1616-3028(20020418)12: 4<266::AID-ADFM266>3.0.CO;2-U.

9. Baum T, Ye S, Uosaki K. Formation of self-assembled monolayers of alkanethiols on GaAs surface with in situ surface activation by ammonium hydroxide. Langmuir. 1999;15:8577-9. https://doi.org/ 10.1021/la991124w.

10. Ye S, Li G, Noda H, Uosaki K, Osawa M. Characterization of selfassembled monolayers of alkanethiol on GaAs surface by contact angle and angle-resolved XPS measurements. Surf Sci. 2003;529: 163-70. https://doi.org/10.1016/S0039-6028(03)00239-5.

11. Ding X, Moumanis K, Dubowski JJ, Tay L, Rowell NL. Fouriertransform infrared and photoluminescence spectroscopies of selfassembled monolayers of long-chain thiols on (001) GaAs. J Appl Phys. 2006. https://doi.org/10.1063/1.2178659.

12. Rei Vilar M, El Beghdadi J, Debontridder F, Artzi R, Naaman R, Ferraria M, et al. Characterization of wet-etched GaAs (100) surfaces. Surf Interface Anal. 2005;37:673-82. https://doi.org/10. 1002/sia.2062.

13. OSAWA M, Ataka K-I, IKEDA M, UCHIHARA H, NANBA R. Surface enhanced infrared absorption spectroscopy. Anal Sci. 1991;7:503-6. https://doi.org/10.2116/analsci.7.Supple_503.

14. Nishikawa Y, Fujiwara K. Surface-enhanced infrared external reflection spectroscopy at low reflective surfaces and its application to surface analysis of semiconductors, glasses, and polymers. Anal Chem. 1993:556-62. https://doi.org/10.1021/ac00053a011.

15. López-Lorente ÁI, Sieger M, Valcárcel M, Mizaikoff B. Infrared attenuated total reflection spectroscopy for the characterization of gold nanoparticles in solution. Anal Chem. 2014;86:783-9. https:// doi.org/10.1021/ac403284f.

16. Wanzenböck HD, Edl-Mizaikoff B, Friedbacher G, Grasserbauer M, Kellner R, Arntzen M, Luyven T, Theiss W, Grosse P (1997) Surface-enhanced infrared absorption spectroscopy (SEIRA) using multireflection ATR-elements. In: Prog. Fourier Transform Spectrosc. Springer Vienna, Vienna, pp 665-667

17. Kellner R, Mizaikoff B, Jakusch M, Wanzenböck HD, Weissenbacher N. Surface-enhanced vibrational spectroscopy: a new tool in chemical IR sensing? Appl Spectrosc. 1997;51:495503. https://doi.org/10.1366/0003702971940774.
18. Ataka K, Heberle J. Biochemical applications of surface-enhanced infrared absorption spectroscopy. Anal Bioanal Chem. 2007;388: 47-54. https://doi.org/10.1007/s00216-006-1071-4.

19. Ataka K, Stripp ST, Heberle J. Surface-enhanced infrared absorption spectroscopy (SEIRAS) to probe monolayers of membrane proteins. Biochim Biophys Acta Biomembr. 2013;1828:2283-93. https://doi.org/10.1016/j.bbamem.2013.04.026.

20. Xu J-Y, Jin B, Zhao Y, Wang K, Xia X-H. In situ monitoring of the DNA hybridization by attenuated total reflection surface-enhanced infrared absorption spectroscopy. Chem Commun. 2012;48:3052. https://doi.org/10.1039/c2cc17058b.

21. Zhang Z, Yoshida N, Imae T, Xue Q, Bai M, Jiang J, et al. A selfassembled monolayer of an alkanoic acid-derivatized porphyrin on gold surface: a structural investigation by surface plasmon resonance, ultraviolet-visible, and infrared spectroscopies. J Colloid Interface Sci. 2001;243:382-7. https://doi.org/10.1006/jcis.2001. 7850 .

22. Gregoriou VG, Rodman SE. Vibrational spectroscopy of thin organic films. In: Griffiths PR, editor. Handb. Vib. Spectrosc. Chichester: John Wiley \& Sons, Ltd; 2006. p. 2670-93.

23. Sato Y, Noda H, Mizutani F, Yamakata A, Osawa M. In situ surfaceenhanced infrared study of hydrogen bond pairing of complementary nucleic acid bases at the electrochemical interface. Anal Chem. 2004;76:5564-9. https://doi.org/10.1021/ac049303s.

24. Bibikova O, Haas J, López-Lorente AI, Popov A, Kinnunen M, Meglinski I, et al. Towards enhanced optical sensor performance: SEIRA and SERS with plasmonic nanostars. Analyst. 2017;142: 951-8. https://doi.org/10.1039/C6AN02596J.

25. EU (2006) Commission Regulation (EC) No. 401/2006. 1-42

26. de Koe WJ. Regulations of the European Union for mycotoxins in foods. Arh Hig Rada Toksikol. 1999;50:37-46.

27. Kang'ethe EK, Lang'a KA. Aflatoxin B1 and M1 contamination of animal feeds and milk from urban centers in Kenya. Afr Health Sci. 2009;9:218-26. https://doi.org/10.4314/ahs.v9i4.52140.

28. Busby W, Wogan G. Aflatoxins. Chem Carcinog. 1984;1971:9451136.

29. Krska R, Schubert-Ullrich P, Molinelli A, Sulyok M, MacDonald S, Crews C. Mycotoxin analysis: an update. Food Addit Contam A. 2008;25:152-63. https://doi.org/10.1080/02652030701765723.

30. Mirghani MES, Che Man YB, Jinap S, Baharin BS, Bakar J. A new method for determining aflatoxins in groundnut and groundnut cake using Fourier transform infrared spectroscopy with attenuated total reflectance. J Am Oil Chem Soc. 2001;78:985-92. https://doi.org/ 10.1007/s11746-001-0376-y.

31. Benzce K, Kiermeier F (1972) Zur Infrarotspektroskopie der Aflatoxine. Mitteilungen aus dem Milchwissenschaftlichen Inst der Tech Univ München, pp 211-214

32. Shamsaie A, Kobarfard F, Yazdanpanah H, Sabzevari O. Is FTIRATR a sensitive tool for determination of aflatoxins? J Am Oil Chem Soc. 2004;81:719-20. https://doi.org/10.1007/s11746-004967-7.

33. Kos G, Sieger M, McMullin D, Zahradnik C, Sulyok M, Öner T, et al. A novel chemometric classification for FTIR spectra of mycotoxin-contaminated maize and peanuts at regulatory limits. Food Addit Contam A. 2016;33:1596-607. https://doi.org/10. 1080/19440049.2016.1217567.

34. Sieger M, Kos G, Sulyok M, Godejohann M, Krska R, Mizaikoff B. Portable infrared laser spectroscopy for on-site mycotoxin analysis. Sci Rep. 2017;7:44028. https://doi.org/10.1038/srep44028.

35. Kos G, Krska R, Lohninger H, Griffiths PR. A comparative study of mid-infrared diffuse reflection (DR) and attenuated total reflection (ATR) spectroscopy for the detection of fungal infection on RWA2corn. Anal Bioanal Chem. 2004;378:159-66. https://doi.org/10. 1007/s00216-003-2245-y.

36. Kos G, Lohninger H, Krska R. Fourier transform mid-infrared spectroscopy with attenuated total reflection (FT-IR/ATR) as a tool for 
the detection of Fusarium fungi on maize. Vib Spectrosc. 2002;29: 115-9. https://doi.org/10.1016/S0924-2031(01)00196-5.

37. Abramović B, Jajić I, Abramović B, Ćosić J, Jurić V. Detection of deoxynivalenol in wheat by fourier transform infrared spectroscopy. Acta Chim Slov. 2007;54:859-67.

38. McMullin D, Mizaikoff B, Krska R. Advancements in IR spectroscopic approaches for the determination of fungal derived contaminations in food crops. Anal Bioanal Chem. 2015;407:653-60. https://doi.org/10.1007/s00216-014-8145-5.

39. INRF (1960) RCA-1 silicon wafer cleaning. 2:2-4.

40. INRF (1960) RCA-2 silicon wafer cleaning. 2:5-7.

41. Schindelin J, Arganda-Carreras I, Frise E, Kaynig V, Longair M, Pietzsch T, et al. Fiji: an open-source platform for biological-image analysis. Nat Methods. 2012;9:676-82. https://doi.org/10.1038/ nmeth.2019.

42. Yuan H, Khoury CG, Hwang H, Wilson CM, Grant GA, Vo-Dinh T. Gold nanostars: surfactant-free synthesis, 3D modelling, and twophoton photoluminescence imaging. Nanotechnology. 2012;23: 075102. https://doi.org/10.1088/0957-4484/23/7/075102.

43. Frens G. Particle size and sol stability in metal colloids. KolloidZeitschrift Zeitschrift für Polym. 1972;250:736-41. https://doi.org/ 10.1007/BF01498565.

44. Frens G. Controlled nucleation for the regulation of the particle size in monodisperse gold suspensions. Nat Phys Sci. 1973;241:20-2. https://doi.org/10.1038/physci241020a0.

45. Garcia M, Blanco JL, Suarez G. Aflatoxins B 1 and G 1 solubility in standard solutions and stability during cold storage. Mycotoxin Res. 1994;10:97-100. https://doi.org/10.1007/BF03192258.
46. Yang CY. Comparative studies on the detoxification of aflatoxins by sodium hypochlorite and commercial bleaches. Appl Microbiol. 1972;24:885-90.

47. Schädle T, Mizaikoff B (2016) Selecting the right tool: comparison of the analytical performance of infrared attenuated total reflection accessories. Appl Spectrosc 0:1-8. doi: https://doi.org/10.1177/ 0003702816641574.

48. Li Y, Wong C (2005) High performance anistropic conductive adhesives for Lead-free interconnects. In: 2005 Conf. High Density Microsyst. Des. Packag. Compon. Fail. Anal. IEEE, pp 1-7

49. Bibikova O, Haas J, López-Lorente ÁI, Popov A, Kinnunen M, Ryabchikov Y, et al. Surface enhanced infrared absorption spectroscopy based on gold nanostars and spherical nanoparticles. Anal Chim Acta. 2017;990:141-9. https://doi.org/10.1016/j.aca.2017. 07.045 .

50. Osawa M (2001) Surface-enhanced infrared absorption. In: Top. Appl. Phys. pp 163-187

51. Wang X, Kim S-S, Roßbach R, Jetter M, Michler P, Mizaikoff B. Ultra-sensitive mid-infrared evanescent field sensors combining thin-film strip waveguides with quantum cascade lasers. Analyst. 2012;137:2322. https://doi.org/10.1039/c1an15787f.

52. Schädle T, Mizaikoff B. Mid-infrared waveguides: a perspective. Appl Spectrosc. 2016;70:1625-38. https://doi.org/10.1177/ 0003702816659668.

Publisher's note Springer Nature remains neutral with regard to jurisdictional claims in published maps and institutional affiliations. 